\title{
Influence of secondary emission yield on the saturation properties of multipactor discharges between two parallel metal plates
}

\author{
M. Buyanova, ${ }^{1}$ V. E. Semenov, ${ }^{1}$ D. Anderson, ${ }^{2}$ M. Lisak, ${ }^{2}$ and J. Puech ${ }^{3}$ \\ ${ }^{1}$ Institute of Applied Physics, Russian Academy of Science, Nizhny Novgorod 603950, Russia \\ ${ }^{2}$ Department of Radio and Space Science, Chalmers University of Technology, \\ Gothenburg SE-412 96, Sweden \\ ${ }^{3}$ Centre National d'Etudes Spatiales, Toulouse 31401, France
}

(Received 7 January 2010; accepted 9 March 2010; published online 8 April 2010)

\begin{abstract}
A detailed numerical simulation analysis is made of the saturation stage of multipactor discharges between two infinite parallel metal plates in vacuum exposed to a rf voltage. The main physical effect causing saturation of the multipactor discharge is increased space charge, as the electron density becomes large. It is found that the properties of the saturation stage depend crucially on the value of the secondary emission yield of the metal surfaces. Below a certain threshold value, the discharge has a two-sided character, but at this threshold the discharge makes an abrupt transition into two decoupled single-sided multipactor discharges containing significantly increased electron densities. The result of the numerical simulations gives a good picture of the saturation properties of the multipactor discharge and is also supplemented by an approximate analytical investigation that highlights and explains the characteristic properties of the observed saturation behavior.
\end{abstract}

(C) 2010 American Institute of Physics. [doi:10.1063/1.3374430]

\section{INTRODUCTION}

The multipactor discharge is an important undesirable effect that occurs in high-power microwave devices operating close to vacuum conditions. It is manifested by an avalanchelike increase in the number of electrons in rf devices when free electrons, accelerated to high energies in a microwave field, hit the walls of the rf device in a periodically repeated process, ${ }^{1,2}$ causing electron multiplication due to secondary emitted electrons. The main undesirable consequences of the multipactor phenomenon are generation of $\mathrm{rf}$ noise, mismatch between rf components due to perturbation of their impedances, and even heating and subsequent damage of the device walls. Accurate predictions of the conditions under which a multipactor discharge may occur are therefore a necessary stage in the design of microwave components. ${ }^{3-8}$ For realistic device geometries, such predictions must be based on numerical simulations. Unfortunately, the secondary emission properties of the device walls are sensitive to contamination of the solid surface and even numerical simulations cannot guarantee multipactor free operation of a device. Thus, when the operation parameters of a system imply a potential risk for a multipactor discharge to develop, a study of the saturation properties of the multipactor becomes important since this latter stage determines the negative consequences of the multipactor discharge. It should be underlined that most measurements of the multipactor characteristics (with the exception of the threshold itself) are carried out after the multipactor has been fully established and a subsequent consistent comparison of the data must be made with theoretical predictions obtained for the multipactor saturation stage.
Multipactor saturation can be caused either by a reduction in the microwave field amplitude due to rf mismatch ${ }^{9,10}$ or by the appearance of a dc electric field in the system due to surface and space charge effects. ${ }^{11-20}$ The pure space charge effect has been considered in investigations of multipactor discharges on metal surfaces, ${ }^{11-15}$ whereas both surface and space charge effects were taken into account when considering multipactor development on dielectric surfaces. ${ }^{16-21}$ The problem of multipactor saturation is too complicated for exact analytical analysis and in order to obtain sufficiently accurate theoretical predictions in a particular case, detailed numerical simulations must be carried out using a particle-in-cell software ${ }^{15}$ or analogous softwares. ${ }^{18,21}$ On the other hand, numerical simulations are inherently restricted to some particular parameter combinations and the subsequent results are not easily extended to other parameter ranges. Therefore different approximate models have also been developed for analytical analysis of the multipactor saturation process.

These models can be divided into two qualitatively different groups. The simplest model is used to estimate the power deposited by the multipactor on the surface of an output microwave transparent window, ${ }^{16,17}$ which is parallel to the microwave electric field. In this case the multipactor avalanche is not associated with any resonance condition and the only effect of the developing dc field on the electron motion is a shortening of the flight time of the electrons before their return to the surface of emission. Within this model, the multipactor avalanche saturates when the secondary electrons no longer acquire sufficiently high energy from the microwave field during their (short) flight times. When the microwave electric field is perpendicular to the surface of emission, growth of the multipactor avalanche is typically associated 
with stable resonant motion of the electrons (both for singlesurface and two-surface multipactor). Therefore the perturbation of the resonance stability by the increasing dc electric field has been considered as the appropriate mechanism for multipactor saturation. ${ }^{11-14,19,20}$

However, it should be noted that possible applications of the latter model to estimate multipactor saturation on metal surfaces are rather restricted. First, the model does not take into account a spread of electron emission velocity which may considerably disturb the multipactor resonance. In addition, the influence of a value of the secondary emission yield (SEY) larger than unity is also an important factor that is ignored within the above model. As was shown in Ref. 22, when the velocity spread is combined with a sufficiently high SEY value, multipactor zones of higher orders may become completely overlapping. This implies that a perturbation of the resonance condition does not necessarily stop the multipactor growth. Specifically, in situations when the multipactor zones are overlapping, perturbations of the resonance can be expected to have a small effect on the multipactor growth and a more probable saturation mechanism is that considered for multipactor discharges on output microwave windows. This means that an increase in the SEY value may result in considerable modification of the multipactor saturation regime, including even the possibility of a transition of the discharge type from two-surface to single-surface multipactor. Such a transition was discussed recently, ${ }^{19,20}$ however, without any relation to the SEY value.

In this paper, a detailed numerical and analytical study is made of the dependence of the properties of the multipactor saturation regime on the SEY value. For better understanding, the simplest geometry is considered, i.e., two parallel metal plates exposed to a monochromatic rf voltage. The numerical simulations have been carried out using a particlein-cell (PIC) software similar to that presented in Ref. 15. The simulations were performed in a wide range of parameters corresponding to different multipactor zones and the saturation levels of the electron density and the power deposition on the metal plates were determined as functions of the SEY value.

Two qualitatively different regimes of multipactor saturation were found. When the SEY value is below a certain threshold, the saturation levels of electron density and power deposition are relatively small. However, these levels increase drastically when the SEY value exceeds a certain threshold value. It is shown that the first saturation regime is associated with the conventional two-sided multipactor, which means that the space charge effect is not strong enough to prevent the electrons from crossing the gap. On the other hand, in the second saturation regime, the multipactor becomes single sided. The transition to the single-sided multipactor is completely caused by the space charge effect which becomes so strong that the majority of the secondary electrons are forced to return to their surface of emission. A similar transition to the single-sided multipactor may also be caused by electric charge accumulation on a dielectric surface under the conditions studied in Ref. 18.

\section{NUMERICAL EXPERIMENTS}

Several series of numerical tests were carried out using a particle-in-cell code to study the saturation effect for different multipactor zones and its dependence on the SEY value. In all series the $\mathrm{rf}$ frequency, $f$, was taken to be $1 \mathrm{GHz}$ and the amplitude of the applied rf voltage was chosen so as to have the same value of the undisturbed rf field amplitude, $E_{0}=1.77 \times 10^{5} \mathrm{~V} / \mathrm{m}$ (i.e., when space charge effects are negligible). This implies that the value of the electron oscillatory velocity is given by $V_{\omega}=e E_{0} /(m \omega) \approx 5 \times 10^{6} \mathrm{~m} / \mathrm{s}$, where $e$ and $m$ denote electron charge and mass, respectively, and $\omega=2 \pi f$ is the angular rf frequency. It was assumed that the electron density was independent of the coordinates along the plate surfaces and only the component of electron motion, normal to these surfaces, was considered. The position of an electron between the plates is described by the coordinate $x$ where the coordinates $x=0$ and $x=L$ correspond to the plate surfaces. The initial phase of the rf voltage was chosen so as to provide an acceleration of the electrons away from the plate corresponding to $x=0$ and toward the plate at $x=L$.

At the beginning of each test 100 seed computer particles were started, being uniformly distributed in the space between the plates, i.e., over the interval $[0 \leq x \leq L]$. Each computer particle represented 100 physical electrons which all had the same parameters of motion. Each computer particle moved under the action of the electric field until a collision with one of the plates occurred. Within the software, the electric field between the plates was recalculated at each time step taking into account the current distribution of the electron space charge density, which was determined by the positions of all computer particles assuming that they fill the cross section with an area equal to $5 \mathrm{~mm}^{2}$. Each collision of a computer particle with a plate was accompanied by the appearance of a number of secondary computer particles (each representing 100 physical electrons) as determined by the SEY value corresponding to the impact energy of a primary electron. A spread over initial electron velocities was also taken into account by assuming the initial velocities of the computer particles (both the seed and the secondary ones) to be described by random values governed by the Maxwellian probability distribution given by Eq. (1) with average value $V_{m}=0.1 V_{\omega}$ and deviation $\Delta V=0.01 V_{\omega}$ (fixed for all tests).

$$
f(V) \propto \exp \left(-\frac{\left(V-V_{m}\right)^{2}}{2 \Delta V^{2}}\right)
$$

The SEY properties were modeled using Vaughan's approximation $^{23}$ for normal impacts, see Eq. (2), with the energy corresponding to the maximum of the secondary emission curve kept fixed, $W_{\max }=350 \mathrm{eV}$, whereas the maximum value of the secondary emission coefficient, $\sigma_{\max }$, was varied from test to test. 


$$
\begin{aligned}
& \sigma(w)=\sigma_{\max } \cdot[w \cdot \exp (1-w)]^{\epsilon}, \\
& \epsilon=\left\{\begin{array}{l}
0.62, \text { if } w<1, \\
0.25, \text { if } w>1,
\end{array}\right. \\
& w=\frac{W-W_{0}}{W_{\max }-W_{0}}, \quad W_{0}=0,
\end{aligned}
$$

where $W$ denotes the impact energy of primary electrons.

In each test series, a detailed study of the dependence of the saturation stage on the SEY value was undertaken for some classical multipactor resonance modes. For this purpose the distance between the plates was kept fixed and so as to approximately satisfy the equality

$$
\lambda \equiv \frac{\omega L}{V_{\omega}}=\pi k \cdot\left(1+\frac{V_{m}}{V_{\omega}}\right),
$$

where $\lambda$ is the normalized gap width ${ }^{24}$ and $k$ is an odd number corresponding to the resonance order (varied between test series). At the initial stage of the multipactor avalanche (when the space charge effect is negligible), such a choice ensures good phase stability of the multipactor resonance with the impact velocity, $V_{\text {impact }} \approx 2 V_{\omega}+V_{m}$, cf. Ref. 24, which corresponds to a SEY value close to the maximum value $\sigma_{\max }$. Each simulation test followed the motion of the electrons until the number of particles saturated around a certain value. The corresponding saturation level of the number of free electrons was then calculated as the average during a large number of rf field cycles in the saturated stage and its dependence on $\sigma_{\max }$ was observed. The power deposition was calculated as deposited energy per rf cycle. In order to verify the assumption that a single-surface regime may appear from a well developed conventional two-sided multipactor near the saturation stage, all computer particle trajectories were traced. Data on all impacts were collected, including impact energy per single electron and flight time, and it was noted whether a computer particle impacted at its surface of emission or at the opposite surface.

The simulation results are presented in Figs. 1-9. Figure 1(a) illustrates the increase in the saturation level of the number of free electrons, $N_{e}$, for increasing SEY values and Fig. 1(b) shows the dependence of the average deposited power, $P_{d}$, on $\sigma_{\max }$ for different resonance modes $k=3, k=5$, and $k=7$. It was found that near the saturation stage, the multipactor development depends strongly on the SEY value. For small SEY values $\left(\sigma_{\max }=1-1.7\right)$, the saturation levels are relatively small and increase continuously for increasing SEY values. This effect is related to the more effective compensation of the electron losses by higher secondary emission. Further increase in the SEY value $\left(\sigma_{\max }=1.75-2.35\right)$ leads to visible small jumps of the saturation levels. It can be assumed that some qualitative changes in the multipactor properties appear at these SEY values. Finally, if the SEY value exceeds a certain threshold value $\left(\sigma_{\max }=2.35-2.4\right)$, the saturation levels of $N_{e}$ and $P_{d}$ increase drastically indicating major changes in the multipactor nature. After this sharp jump, further increase in the SEY coefficient only re-
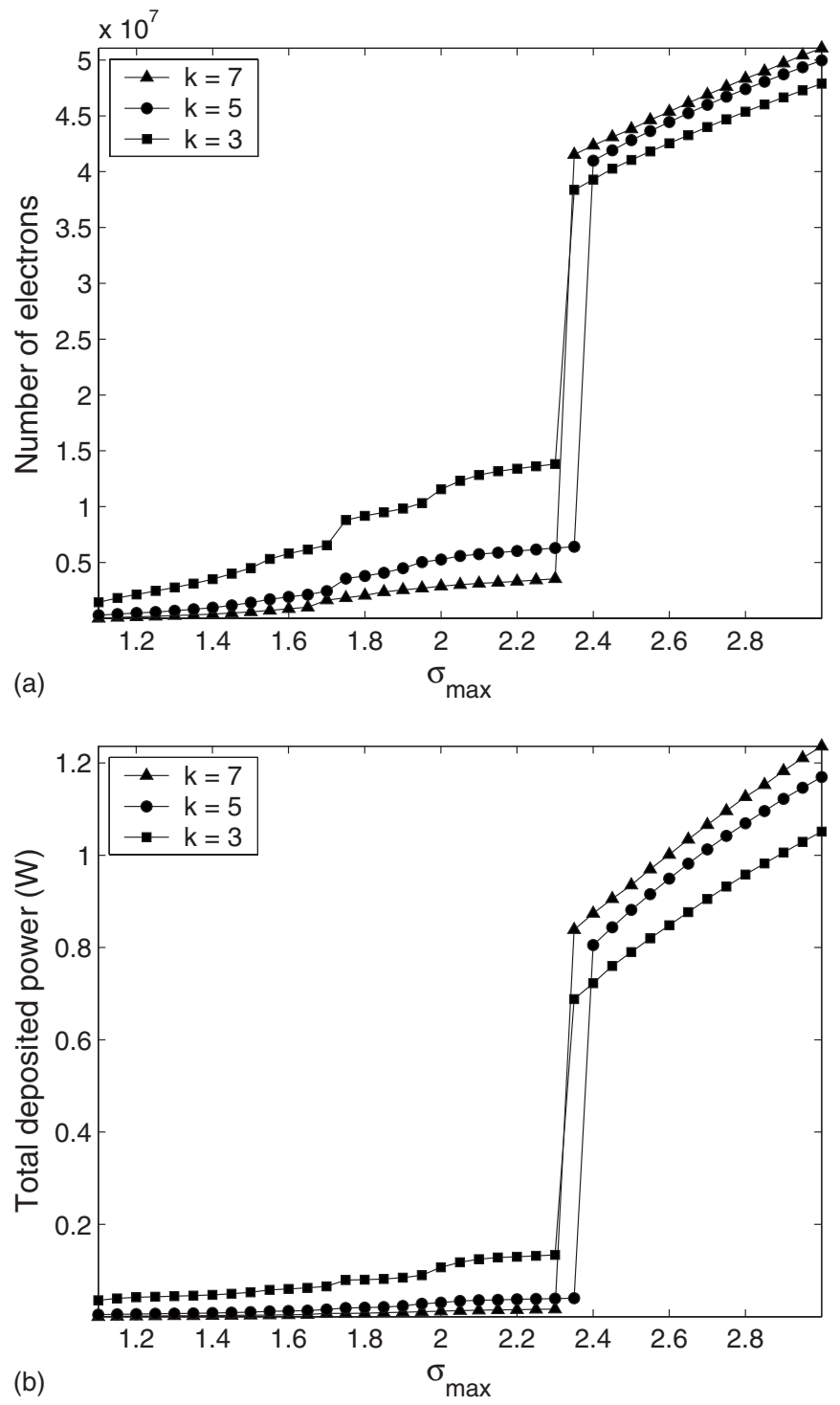

FIG. 1. Saturation levels of the number of electrons (a) and of the total power deposited on the surfaces (b) per $5 \mathrm{~mm}^{2}$ of plate area as functions of $\sigma_{\max }$. Lines marked with squares correspond to the resonance mode with $k=3(\lambda=10.37)$, circles to the resonance mode with $k=5(\lambda=17.28)$, and triangles to the resonance mode with $k=7(\lambda=24.19)$.

sults in a gradual rise of the levels, similar to that at low SEY values and also caused by compensation of electron losses.

Classical resonance theory predicts that for increasing resonance order, the resonance stability factor should decrease. Therefore one expects smaller saturation levels for the higher order resonances, if the saturation is caused by a perturbation of the stability due to the space charge effect. This is completely confirmed by the simulation results for small SEY values. However, the situation is very different for high SEY values, which indicates that another saturation mechanism is dominating.

The dynamical properties of the multipactor were studied in detail for different values of $\sigma_{\max }$. Figures 2(a)-2(c) show the time evolution of the number of particles and of the power deposited on the surfaces during the initial stage of the avalanche growth. The strong oscillations in the curves describing the deposited power [especially pronounced in 

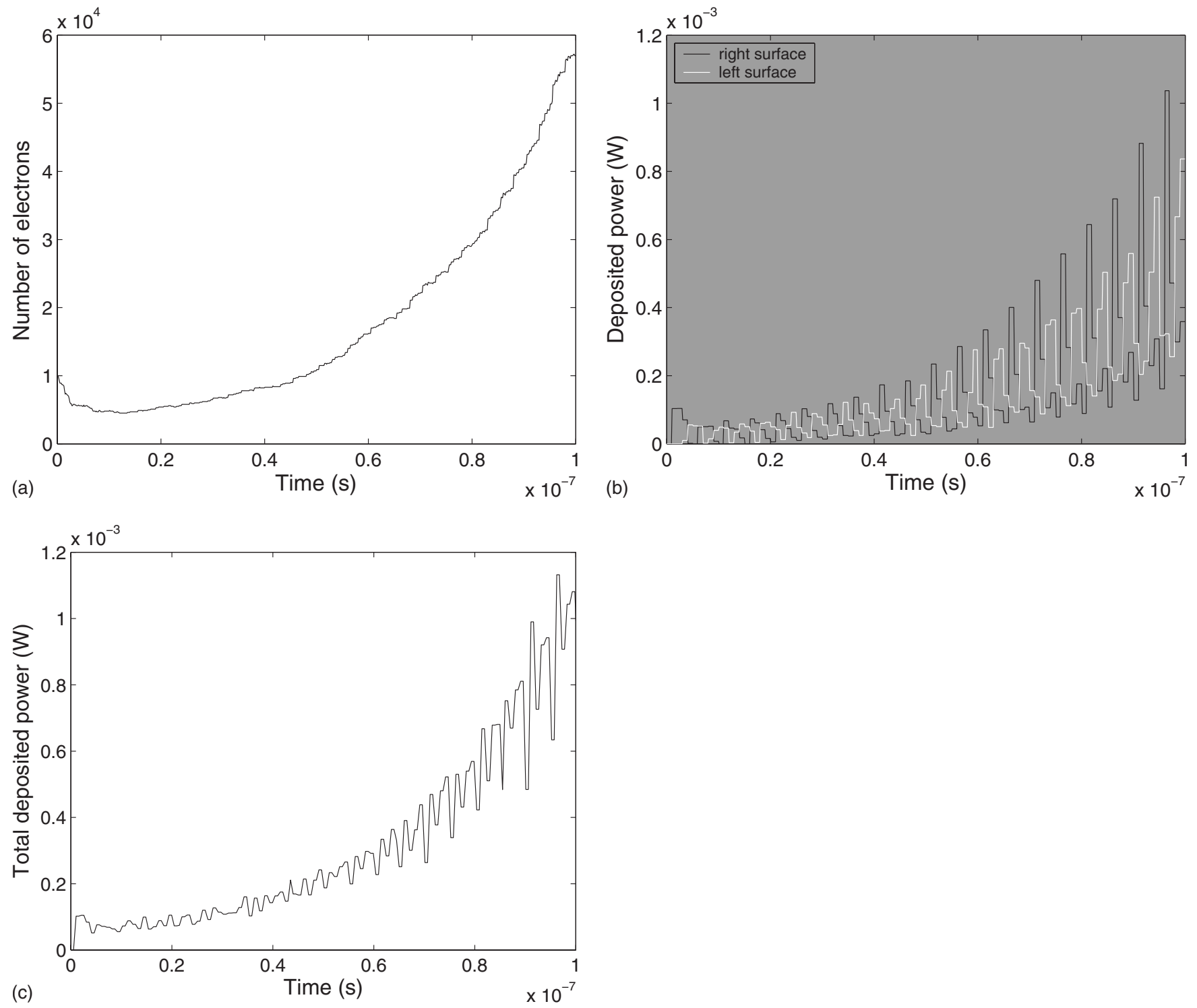

FIG. 2. Time evolution of the number of electrons (a) and of the deposited power (b), (c) per $5 \mathrm{~mm}^{2}$ of plate area during the initial stage of the avalanche growth. Classical mode $k=5(\lambda=17.28)$ and $\sigma_{\max }=1.1$. In (b) the white line corresponds to the surface at $x=0$ and the black line to the surface at $x=L$.

Fig. 2(b)] are caused by a concentration of the electrons into thin layers oscillating in phase with the rf field-a feature typical of stable multipactor resonance.

Figure 3 shows the impact statistics during the initial stage of the multipactor development (calculated at $10^{-7} \mathrm{~s}$ after the start of the simulation). Each dot in Fig. 3 represents a secondary electron trajectory. White dots stand for trajectories that end at the same surface as they start, while black dots represent trajectories that end at the surface opposite to the starting surface. The trajectories shown in Fig. 3 correspond to $N_{c p}$ recent impacts of computer particles; this number can be obtained from the number of electrons $N$ shown in Fig. 2(a) according to $N_{c p}=N / 100$. As is seen from Fig. 3, most of the impacts are registered as occurring on the surface opposite to the surface of emission. During each period, the majority of trajectories that allow electron multiplication are two sided. Therefore it can be concluded that the multipactor development regime is essentially two sided. In Fig. 3, in agreement with the resonance theory, the majority of impacts are concentrated around a flight time equal to $2.5 \mathrm{rf}$ periods and the corresponding impact velocity is close to $2 V_{\omega}+V_{m}$, i.e., an impact energy close to $W_{\max }=350 \mathrm{eV}$, which ensures effective electron multiplication. The observed deviations from the resonance are caused by the small spread of the electron initial velocity. The few white dots close to the left bottom corner represent a small fraction of particles which are thrown out of resonance due to the small spread of the electron initial velocity.

After many rf cycles, the electric field produced by the large number of particles in the avalanche becomes strong enough to defocus and disperse the electron cloud and saturation is reached. Figures $4(\mathrm{a})$ and 4 (b) illustrate the time evolution of the number of particles and the power deposited on the surfaces at saturation. Due to Coulomb repulsion, the spatial distribution of the electron cloud is broadened and this results in a spread of impact phases, which explains why the power deposition curve is smoother than that during the initial stage of the multipactor development [cf. Fig. 2(c)]. 


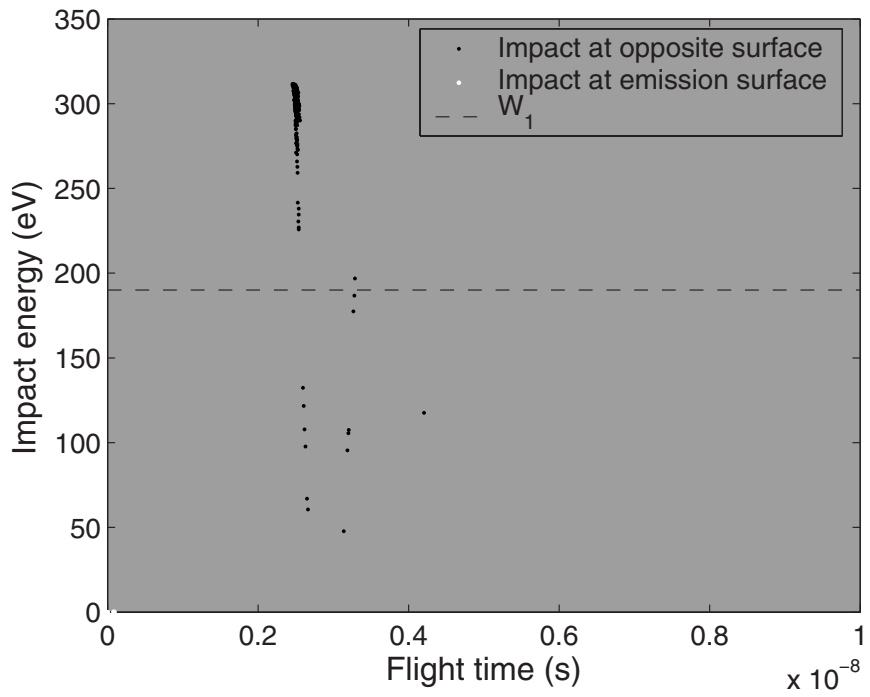

FIG. 3. Impact energies and flight times typical of electrons crossing the gap (black dots) and returning to the surface of emission (white dots) during the initial stage of the avalanche growth. Classical mode $k=5 \quad(\lambda=17.28)$ and $\sigma_{\max }=1.1$. The black dashed line indicates first cross over point energy $W_{1}$ calculated for $\sigma_{\max }=1.1$ and given position of the maximum of the secondary emission curve.

Also due to the electron repulsion at saturation, the cloud has a "tail" of strongly retarded electrons. This tail can be seen in the impact statistics in Fig. 5, obtained at the end of the numerical test [at $5 \times 0^{-7} \mathrm{~s}$ after the start of the simulation shown in Figs. 4(a) and 4(b)].

Similarly to Fig. 3, Fig. 5 shows different secondary electron trajectories corresponding to $N_{c p}$ recent impacts of computer particles [where $N_{c p}$ can be obtained from the number of electrons $N$ shown in Fig. 4(a) as $\left.N_{c p}=N / 100\right]$. However, unlike Fig. 3, Fig. 5 describes the statistical characteristics of the ensemble of electrons at saturation. As shown in Fig. 5, part of the tail electrons crosses the gap, but has considerably longer flight time compared to the $2.5 \mathrm{rf}$ cycles being typical of the original resonance mode. Another part of the retarded electrons return to their surface of emission although their flight time is not so short as in the case when the electron starts with the wrong rf phase and the rf field pushes it back almost immediately. The impact energy of these returning electrons is not very small and they could give rise to development of a single-surface multipactor. However, in the considered case of small $\sigma_{\max }$, the impact energy of the returning electrons is less than the first crossover energy of the SEY curve. The ratio of the number of trajectories that cross the gap between the surfaces to the number of those that return to the surface of emission is practically constant at the saturation stage (small oscillations may be present). Since the majority of those trajectories that allow electron multiplication are still two sided, it is possible to conclude that the multipactor remains in the conventional two-sided regime also during the saturation stage.

The features observed for increasing values of $\sigma_{\max }$ provide support for a transition to new multipactor regimes, including the single-surface one. Figures 6(a) and 6(b) illustrate the time evolution of the number of particles and the power deposited on the surfaces at saturation for a higher
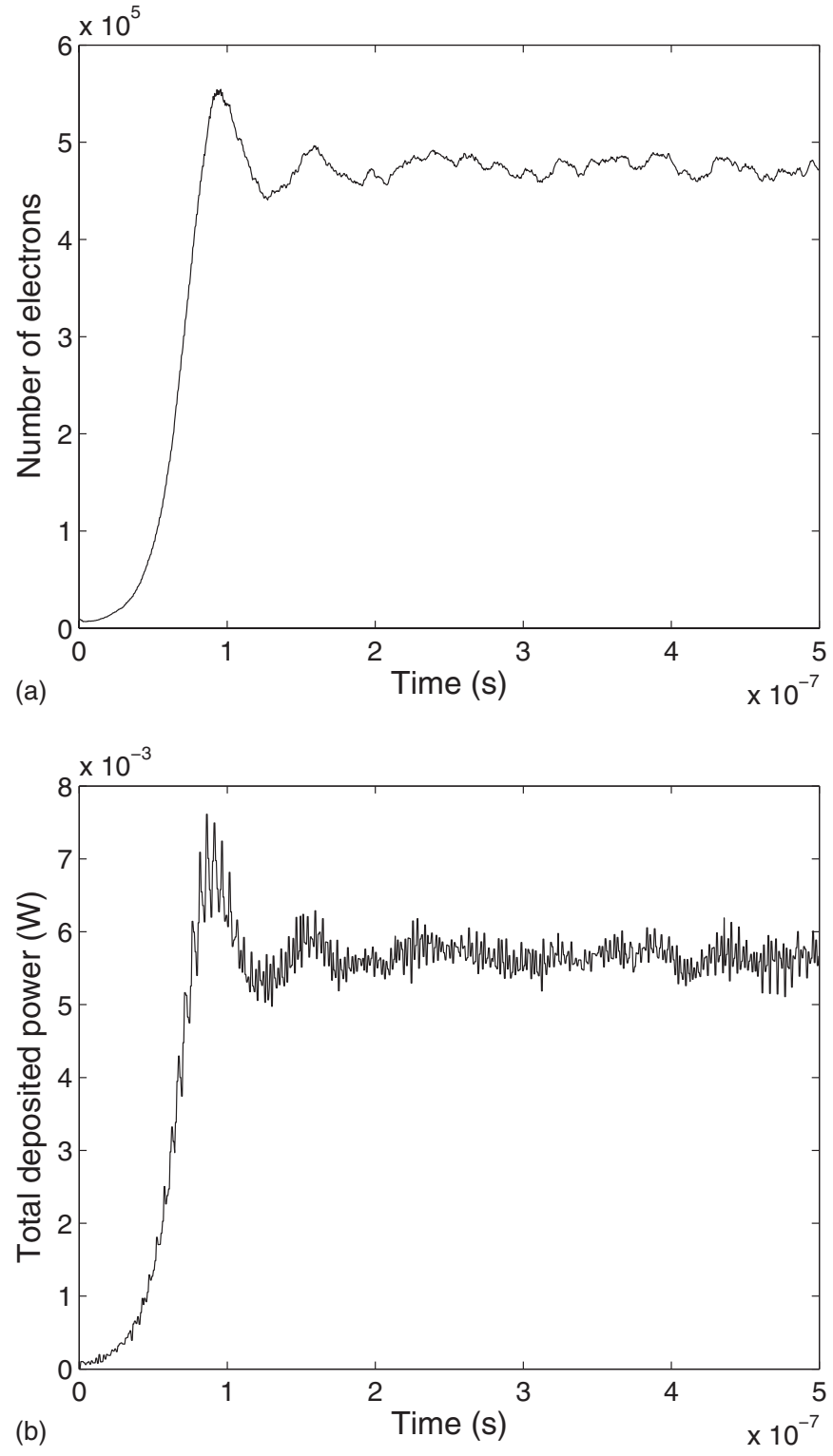

FIG. 4. Time evolution of the number of electrons (a) and of the total deposited power (b) per $5 \mathrm{~mm}^{2}$ of plate area during approach to saturation. The resonance mode order is $k=5(\lambda=17.28)$ and $\sigma_{\max }=1.2$.

value of the SEY. Figure 7 shows the impact statistics obtained at $5 \times 10^{-7} \mathrm{~s}$ after the start of the simulation. Like the previous figures of this type, Fig. 7 shows recently impacted secondary electron trajectories at the saturation stage. It is seen that a large number of those trajectories that return to the surface of emission are present during all $\mathrm{rf}$ periods at saturation and that resonant electron multiplication is possible for a fraction of these single-sided trajectories. Therefore it is possible to conclude that a number of single-surface resonant trajectories become established during the approach to saturation. A typical feature for such regimes is that the electron flight times $\left(1 \times 10^{-9}, 2 \times 10^{-9}, 3 \times 10^{-9} \mathrm{~s}\right.$, etc. equal an integer number of rf cycles for the single-surface regime and the impact energies are larger than $W_{1} \cdot{ }^{25}$ Higher order single-surface regimes appear first. However, these modes are possible only if the static (space charge) electric field returning electrons to the surface of emission is not too 


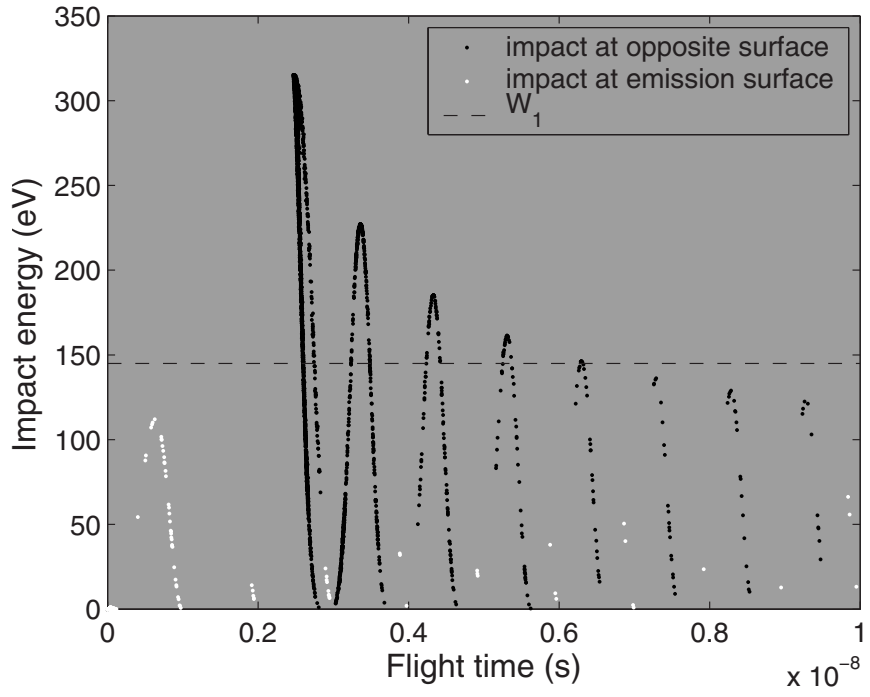

FIG. 5. Impact energies and flight times typical of electrons crossing the gap (black dots) and returning to the surface of emission (white dots) during approach to saturation. Resonance mode $k=5(\lambda=17.28), \sigma_{\max }=1.2$. The black dashed line indicates the first cross over point energy $W_{1}$, calculated for $\sigma_{\max }=1.2$ and given position of the maximum of the secondary emission curve.

strong. The lower order modes appear consecutively as the number of particles and the static electric field increase. Saturation is reached when the lowest single-surface regime, with an electron flight time equal to the rf field cycle $\left(1 \times 10^{-9}\right.$ seconds for the given parameters), has developed. For the given value of $W_{\max }=350 \mathrm{eV}$, this occurs for values of $\sigma_{\max }$ larger than approximately 1.75. The realization of the single-surface regimes leads to visible small jumps in the saturation levels of $N_{e}$ and $P_{d}$ [see Figs. 1(a) and 1(b)]. Nevertheless, the two-sided multipactor regimes still dominate and the saturation properties of the avalanche are still determined mainly by the characteristics of the electron motion of the two-sided multipactor.

However, as the SEY value increases, the situation changes. When $\sigma_{\max }$ exceeds a certain threshold value, the saturation levels of $N_{e}$ and $P_{d}$ rise sharply. Figures 8(a), 8(b), and 9 illustrate how single-surface regimes, originating from the conventional two-sided regime corresponding to $k=5$, develop under the action of the strong space charge effect and become dominant at the saturation stage for $\sigma_{\max }$ higher than 2.35-2.4. Like in previous similar figures, Fig. 9 shows trajectories of recently impacted secondary electrons at saturation. It is seen that the majority of the trajectories return to the surface of emission and resonant electron multiplication is possible for a large fraction of these trajectories. At the same time only a small fraction of the trajectories are two sided (black dots). The electric field produced by the powerful single-surface regimes suppress the resonance and energy conditions for the two-sided regimes so that the majority of the electrons can no longer cross the gap or gain sufficient energy during the transit as shown in Fig. 9. In this case, almost a complete change in multipactor type is observed when approaching the saturation stage. The most powerful component among the single-surface regimes is the first
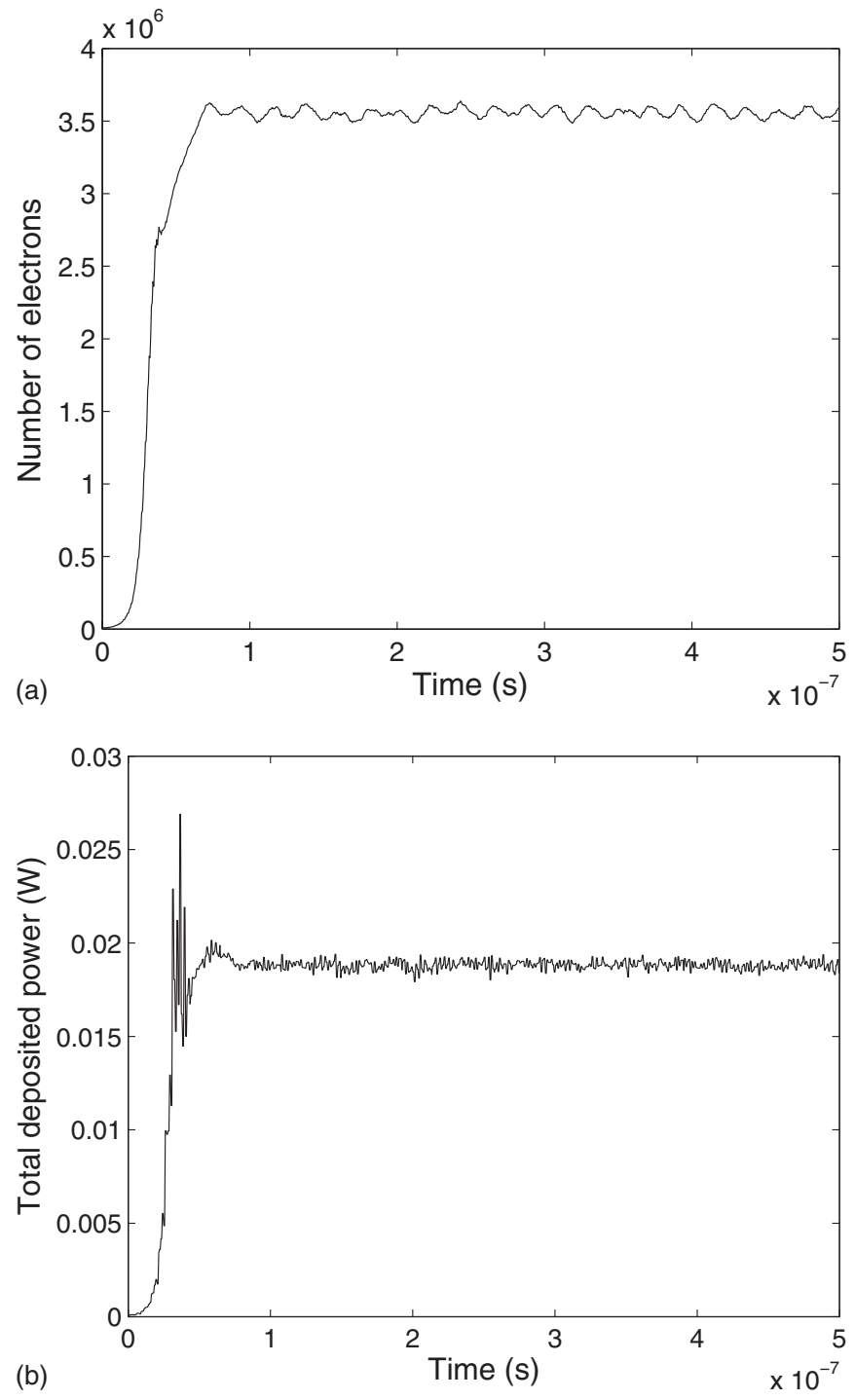

FIG. 6. Time evolution of the number of electrons (a) and of the total deposited power (b) during approach to saturation. Classical mode $k=5$ $(\lambda=17.28)$ and $\sigma_{\max }=1.75$.

resonant mode for which the electron flight time is equal to the rf cycle.

This change in multipactor type is accompanied by a drastic increase in the saturation levels of $N_{e}$ and $P_{d}$. This can be explained by the fact that the single-surface regime, which dominates during the approach to saturation, both provides higher avalanche growth rate than the original classical mode (due to much shorter electron flight time) and is more stable against the perturbations which tends to disturb the resonance. It is also interesting to note that the first classical two-sided mode $k=1$ does not transform completely into a single surface mode at high SEY values. This is due to the fact that the electron flight time in the case of the first singlesurface regime is two times larger than that in the first twosided regime. Consequently, the single surface regime develops more slowly. It also seems reasonable that the perturbations of the electron motion, which tend to suppress the resonance, accumulate to larger values for longer flights, e.g., we can expect that the first two-sided mode should be more resistant to perturbations than the first single-surface 


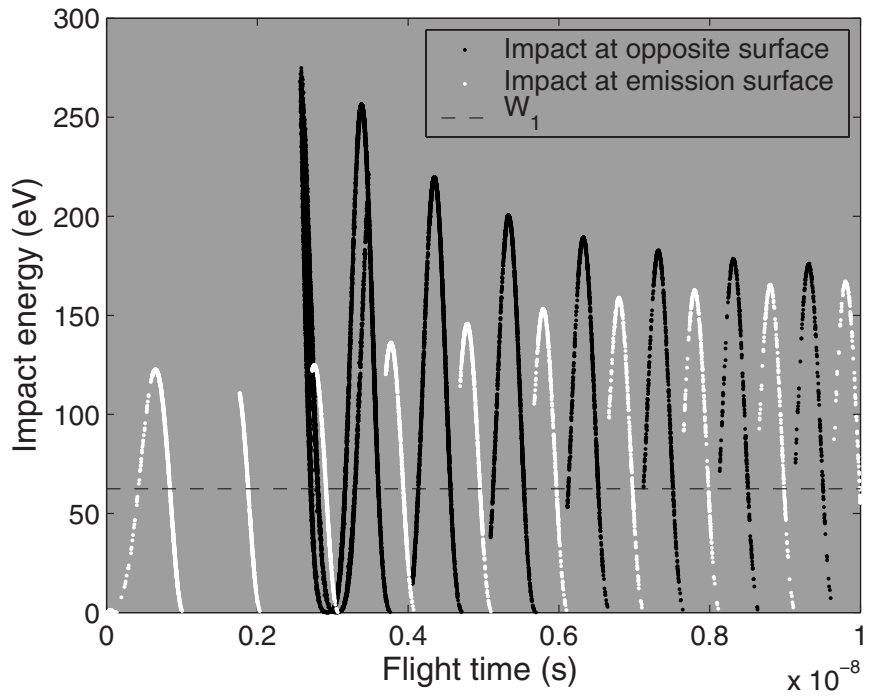

FIG. 7. Impact energies and flight times typical of electrons crossing the gap (black dots) and returning to the surface of emission (white dots) during approach to saturation. Classical mode $k=5(\lambda=17.28)$ and $\sigma_{\max }=1.75$. The black dashed line indicates the first cross over point energy $W_{1}$ calculated for $\sigma_{\max }=1.75$ and given position of the maximum of the secondary emission curve.

mode. Therefore the originally two-sided classical mode $k$ $=1$ remains mostly two-sided at high SEY values, although an extra single-surface component also appears during the approach to saturation.

Finally, in order to verify that the coefficient of secondary emission determines the changes in multipactor character, additional numerical simulations were carried out using a stepwise approximation model of the variation of the SEY with impact energy:

$$
\sigma=\sigma_{\max }, \quad \text { if } \quad W_{1} \leq W \leq W_{2}, \quad \text { else } \quad \sigma=0 .
$$

In this model, the first cross over point energy $W_{1}$ is fixed and equal to $53 \mathrm{eV}\left(W_{2}=10000 \mathrm{eV}\right)$ and only the SEY coefficient $\sigma_{\max }$ is varied. In numerical simulations carried out for the classical mode $k=5$, results similar to the previous ones were observed, viz., an increase in the saturation levels of $N_{e}$ and $P_{d}$ corresponding to the appearance of new multipactor regimes [Figs. 10(a) and 10(b)]. The result clearly demonstrates that changes in the multipactor type are not caused by the shift of the first cross over point $W_{1}$ toward lower energies (which would result from increasing $\sigma_{\max }$ with fixed $W_{\max }$ ), but is a consequence of the increasing SEY coefficient. However, the typical threshold values of $\sigma_{\max }$ are indeed strongly affected by the position of the first cross over point.

\section{DISCUSSION}

It is interesting to compare the above simulation results with the predictions of simple theories developed previously for estimating the parameters of the saturated multipactor discharge. Following Refs. 11 and 12 we first consider a perturbation of the stability of the two-sided multipactor resonance and then present estimates of the conditions under
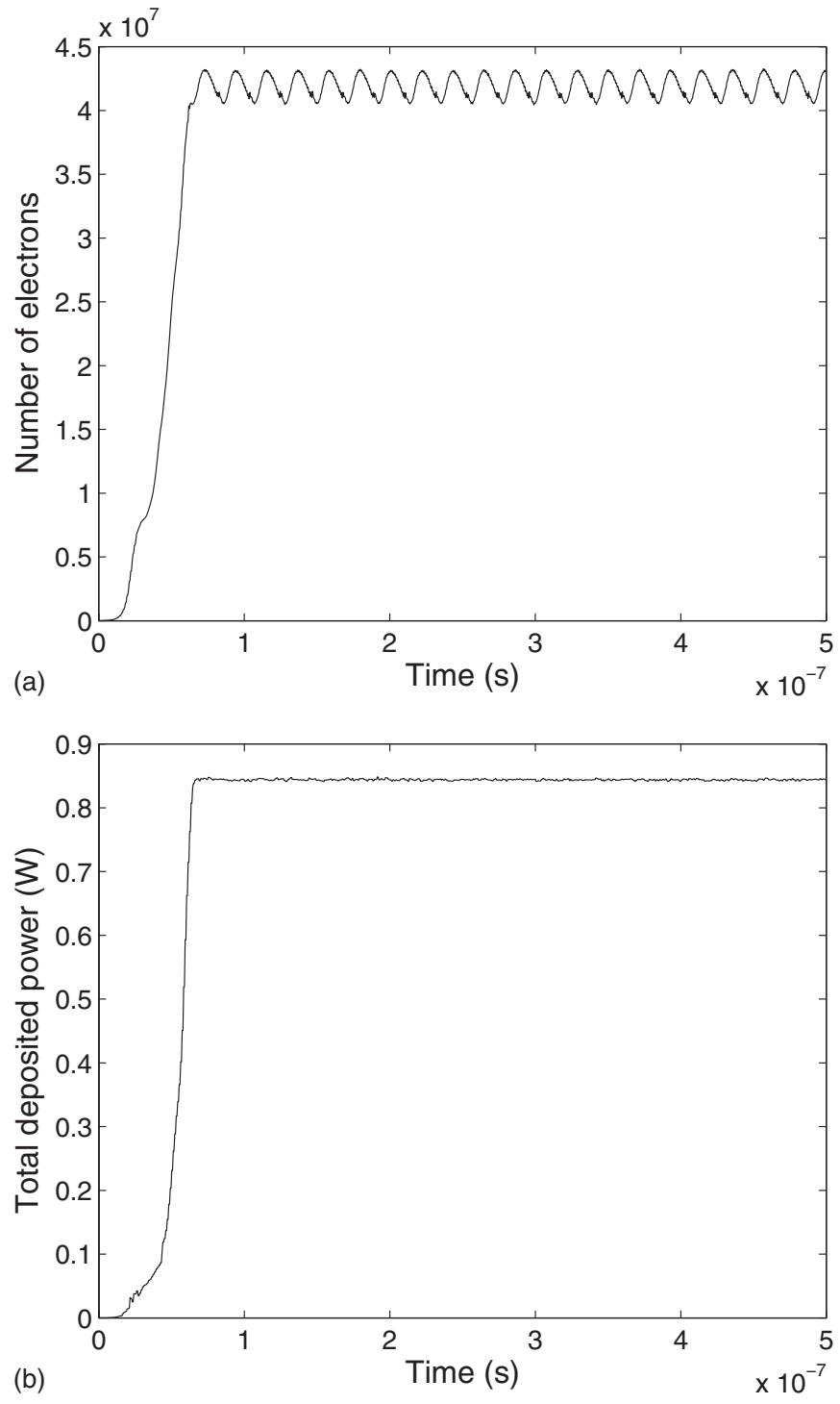

FIG. 8. Time evolution of the number of electrons (a) and of the total deposited power (b) during approach to saturation. Classical mode $k=5$ $(\lambda=17.28)$ and $\sigma_{\max }=2.45$.

which the single surface multipactor ${ }^{19,20}$ occurs. The analytical analysis of the perturbation of the multipactor resonance is based on the following normalized variables: ${ }^{24}$

$$
\xi=\frac{x \omega}{V_{\omega}}, \quad \phi=\omega t=2 \pi f t .
$$

These parameters are convenient since the resonance mode of a particular order, $k$, can easily be identified by its value of the normalized gap width $\lambda$ [cf. Eq. (3)]. Under the conditions of a classical multipactor resonance, the growth of the electron avalanche is associated with the picture of a thin electron cloud oscillating between the metal plates in phase with the rf field. Consequently only the motion of the center of the cloud is considered when studying the initial stage of the multipactor. However, in order to study the saturation stage, one must use a more sophisticated approximation that distinguishes the motion of the center of the cloud from the motion of its front and tail parts. Assuming the total electric field in the space between the plates to be a superposition of 


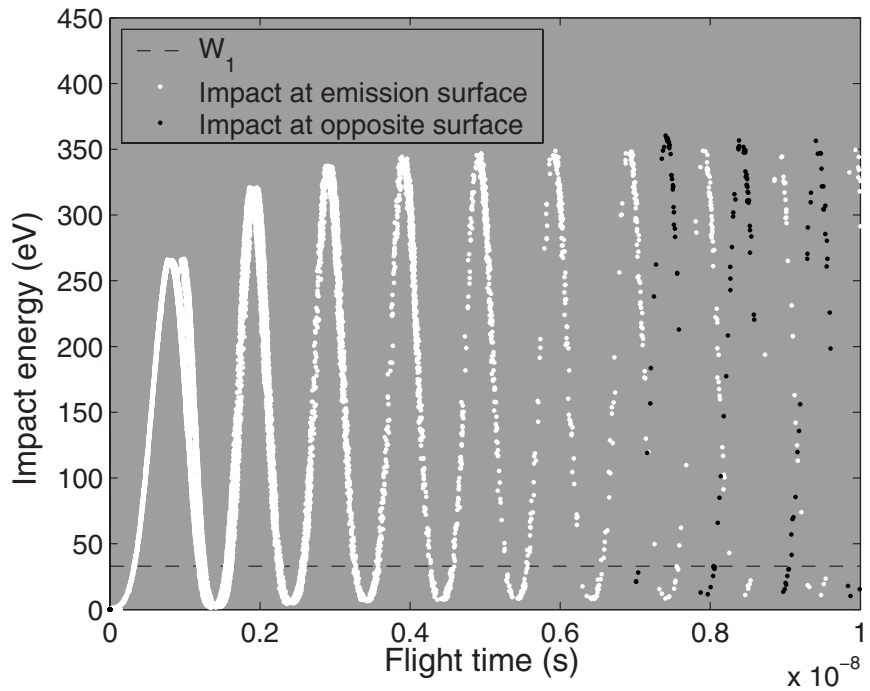

FIG. 9. Impact energies and flight times typical of electrons crossing the gap (black dots) and returning to the surface of emission (white dots) during approach to saturation. Classical mode $k=5(\lambda=17.28)$ and $\sigma_{\max }=2.45$. The black dashed line indicates first cross over point energy $W_{1}$, calculated for $\sigma_{\max }=2.45$ and given position of the maximum of the secondary emission curve.

the rf electric field of fixed amplitude $E_{0}$ and the static electric field, $E_{s c}$, produced by the electron cloud, the equations of motion can be presented in the following forms for the different parts of the cloud:

$$
\begin{aligned}
& \mathrm{F}: \frac{d^{2} \xi}{d \phi^{2}}=\sin \phi+\varepsilon, \\
& \mathrm{R}: \frac{d^{2} \xi}{d \phi^{2}}=\sin \phi, \\
& \mathrm{T}: \frac{d^{2} \xi}{d \phi^{2}}=\sin \phi-\varepsilon,
\end{aligned}
$$

where $\varepsilon=E_{s c} / E_{0}$ and the notations $F, R$, and $T$ indicate parameters of the front, center, and tail parts of the cloud, respectively. In the above equations it is assumed that the motion of the center of the cloud is not affected by the developing space charge, whereas the front and tail parts get additional acceleration or retardation as a result of the Coulomb repulsion between the electrons. A more accurate modeling of the space charge induced disturbance of the motion of the cloud center was made in Ref. 11. Although the present simplified model cannot be used to make accurate predictions, it should make it possible to understand the main qualitative features observed in the numerical simulations. The value $E_{s c}$ is related to the total charge density, $\rho$, in the cloud according to $E_{s c}=\rho /\left(2 \epsilon_{0}\right)$, where $\epsilon_{0}$ is the dielectric constant in vacuum.

Assuming all electrons to start with the same initial velocity, $V_{0}$, Eqs. (6)-(8) determine the relations between the starting phases and the normalized gap width, $\lambda \equiv \omega L / V_{\omega}$, which are necessary to make possible resonant motion with a flight time equal to an odd number, $k$, of half rf cycles:
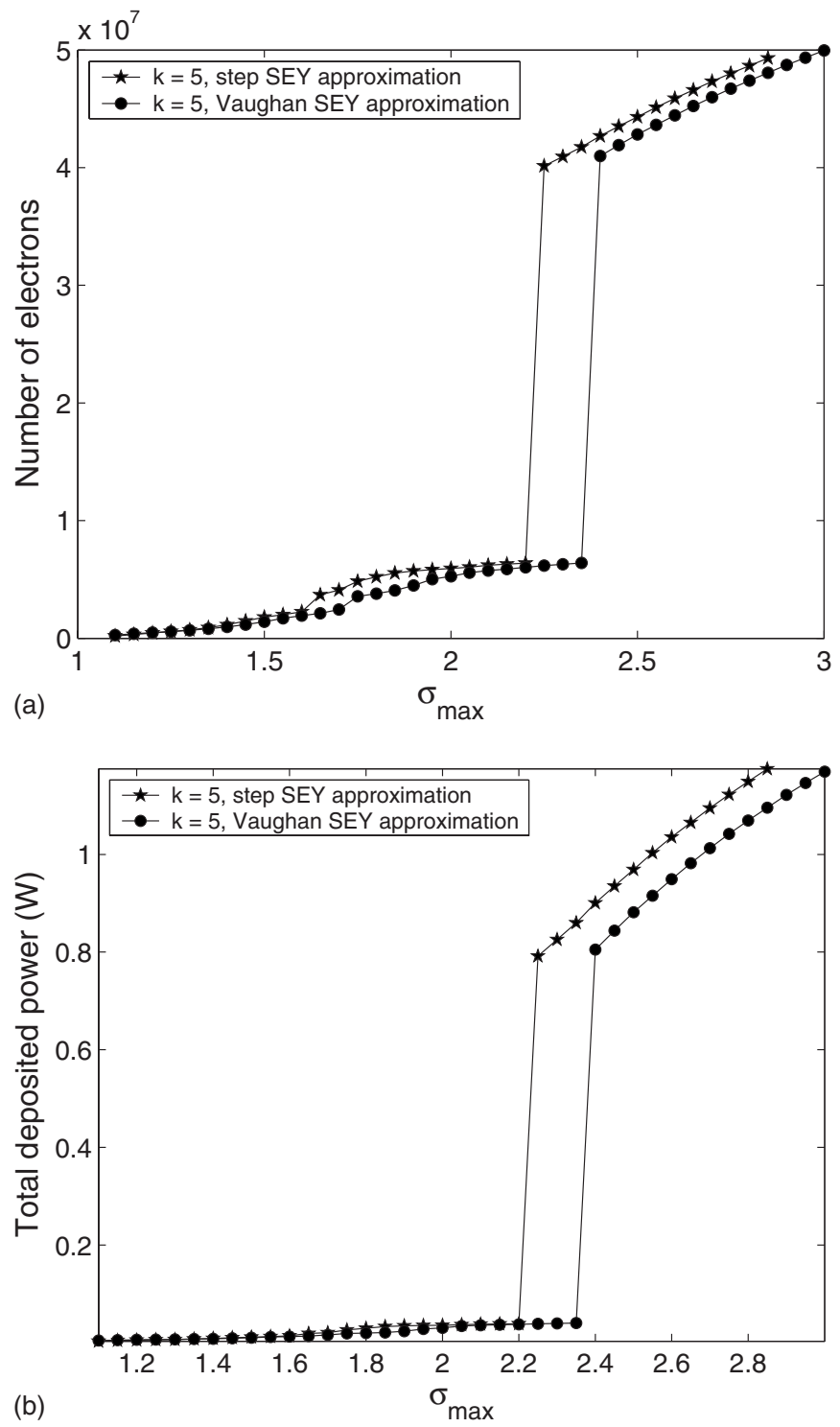

FIG. 10. Saturation levels of the number of electrons (a) and of the total power deposited on the surfaces (b) as functions of $\sigma_{\max }$. Classical mode $k=5(\lambda=17.28)$. Lines marked with circles correspond to simulations carried out using the SEY approximation given by Eq. (2) and lines marked with stars to simulations carried out for the SEY approximation given by Eq. (4).

$$
\begin{aligned}
& \mathrm{F}: \lambda=T\left(v_{0}+\cos \phi_{F}\right)+2 \sin \phi_{F}+\varepsilon T^{2} / 2, \\
& \mathrm{R}: \lambda=T\left(v_{0}+\cos \phi_{R}\right)+2 \sin \phi_{R}, \\
& \mathrm{~T}: \lambda=T\left(v_{0}+\cos \phi_{T}\right)+2 \sin \phi_{T}-\varepsilon T^{2} / 2,
\end{aligned}
$$

where $v_{0}=V_{0} / V_{\omega}$ denotes the normalized initial velocity of an electron, $T=\pi k$ is the normalized resonant electron flight time, and $\phi_{F}, \phi_{R}$, and $\phi_{T}$ denote the resonant starting phases for the different parts of the electron cloud. It should be emphasized that such resonances are possible only for the case of $\sigma=1$ and $\varepsilon=$ const. Considering small deviations of the starting phases from their resonant values, one can determine the parameters regions where the resonances are stable for all parts of the electron cloud.

The fact that the stability of the resonant motion is achieved simultaneously for the $F, R$, and $T$ electrons in the 
case of $\sigma=1$ indicates a balance between the self-focusing of the electron cloud under the conditions of a classical resonance and the space charge effect that tends to defocus the cloud. The outermost resonance phases $\phi_{F}$ and $\phi_{T}$ determine a finite width of the electron cloud. As the space charge effect becomes stronger (i.e., higher $\varepsilon$ values), the interval between $\phi_{F}$ and $\phi_{T}$ increases. However, there exists a threshold maximum value of $\varepsilon$ for the widening of the cloud and, when this threshold is exceeded, electron losses start to occur. An estimate of the threshold value of $\varepsilon$ can be obtained from the stability conditions for the resonant phases of the $F$, $R$, and $T$ electrons. Specifically, in the case of relatively small initial velocity $\left(v_{0} \ll 1\right)$ for the $R$ electrons, the stability conditions for a high order resonance can be expressed in the explicit form ${ }^{24}$

$$
-\frac{2}{T}<\phi_{R}<\frac{2}{T}, \quad-\frac{6}{T}<\lambda-T\left(1+v_{0}\right)<\frac{2}{T} .
$$

A similar analysis shows that a stable resonance of the $R$ electrons is accompanied by a stable resonance of the $F$ and $T$ electrons, if the space charge effect is sufficiently weak and Eq. (12) is consistent with the following analogous conditions for the front and tail parts of the electron cloud:

$$
\begin{gathered}
-\frac{2}{T}<\phi_{F}<\frac{2}{T}, \quad-\frac{6}{T}<\lambda-T\left(1+v_{0}\right)-\frac{\varepsilon T^{2}}{2}<\frac{2}{T}, \\
-\frac{2}{T}<\phi_{T}<\frac{2}{T}, \quad-\frac{6}{T}<\lambda-T\left(1+v_{0}\right)+\frac{\varepsilon T^{2}}{2}<\frac{2}{T} .
\end{gathered}
$$

For example, when $\phi_{R}=0$ [i.e., when $\lambda=T\left(v_{0}+1\right)$ ], stable resonant motion of the $F$ electrons is possible as long as $\varepsilon<12 / T^{3}$, whereas the possibility of stable resonant motion of the $T$ electrons requires the more strict inequality $\varepsilon$ $<4 / T^{3}$. This implies that the presence of the space charge cloud does not destroy the stable resonant motion of any electron in the cloud as long as the parameter $\varepsilon$ is less than the minimum of the above thresholds. In fact, an increase only results in a shift of the resonant phases $\phi_{F}$ and $\phi_{T}$ which determine the finite width of the electron cloud, but does not lead to any electron losses. Finally, it should be emphasized that under the conditions of multipactor resonance, any SEY value larger than unity results in an electron avalanche which saturates by the space charge effect only after a finite threshold in electron number is reached. A reasonable estimate of this threshold can be obtained from the equality:

$$
\varepsilon_{1}=4 / T^{3},
$$

using the concept of a thin charged layer within which the following relation is valid between the number of multipacting electrons and the $\varepsilon$ value,

$$
N=\frac{2 S E_{0} \varepsilon \epsilon_{0}}{e},
$$

where $S$ denotes the area of the surfaces.

The results of the numerical simulations show that the saturation level of the multipactor is in good agreement with the simple estimate given by Eqs. (15) and (16) when the SEY value is marginally larger than unity $\left(\sigma_{\max } \leq 1.1\right)$, see
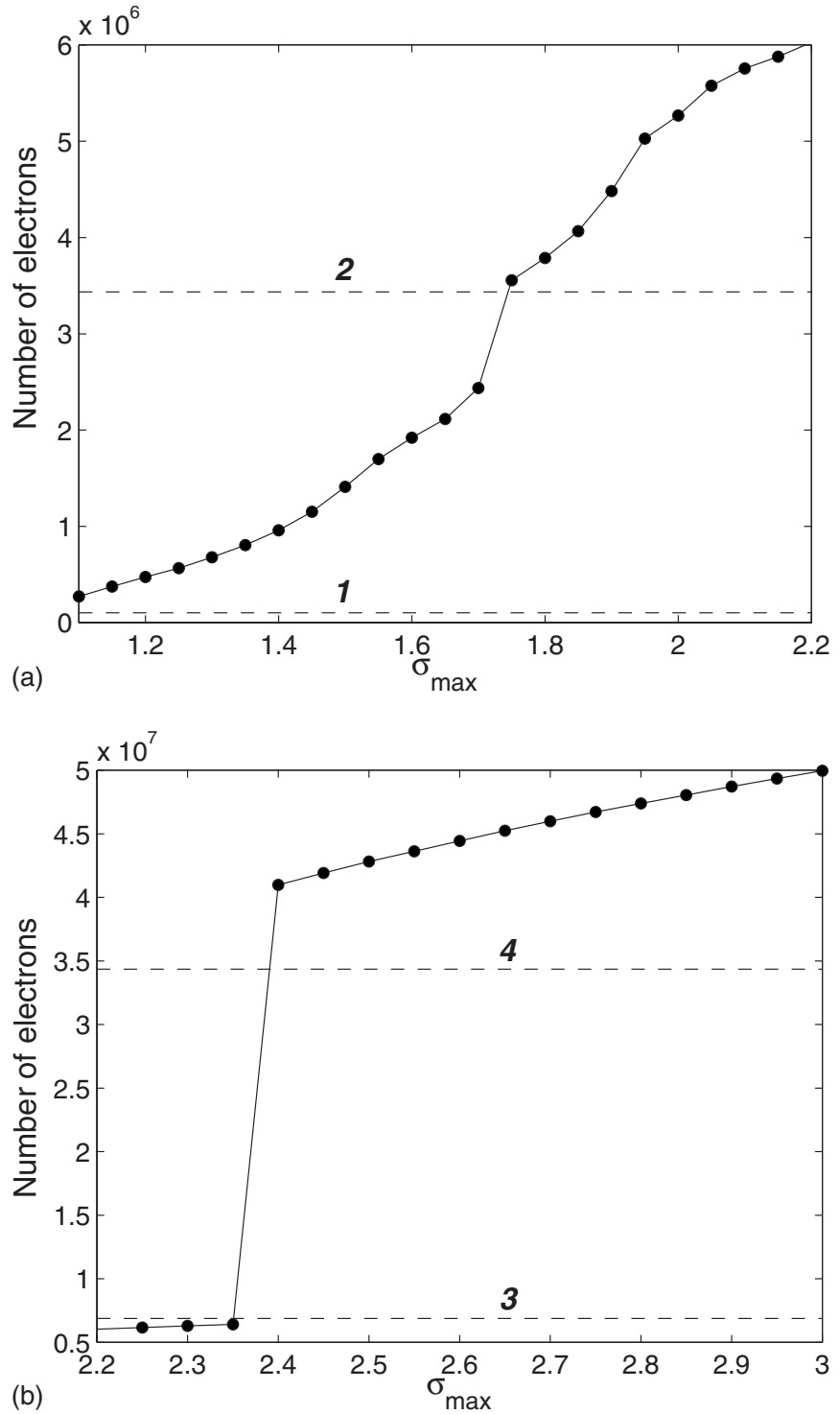

FIG. 11. Saturation level of the number of electrons as function of $\sigma_{\max }$ for the classical resonance mode $k=5(\lambda=17.28)$ in the ranges $1.1<\sigma_{\max }$ $<2.2$ (a) and $2.2<\sigma_{\max }<3$ (b). The solid line marked with circles corresponds to the results of numerical PIC simulations. The dashed lines correspond to the theoretical estimates obtained using Eq. (16). Line 1 corresponds to the estimate $\varepsilon_{1}$ from Eq. (15), line 2 corresponds to the estimate $\varepsilon_{2}$ from Eq. (18), line 3 corresponds to the estimate $2 \varepsilon_{2}$ with $\varepsilon_{2}$ determined from Eq. (18), and line 4 corresponds to the estimate $\varepsilon_{3}$ from Eq. (20).

Fig. 11. However, an analysis of the resonance stability cannot predict the growth of the saturation level with increasing secondary emission yield. ${ }^{11,12}$ To understand this growth one must take into account that the evolution of the width of the electron cloud depends on the evolution of the electron number. A more accurate model has to include the simultaneous increase in both these parameters, especially when the SEY value is well above unity, since this growth continues until the outermost electrons in the cloud reach an unfavorable rf phase at the moment of their collision with one of the plates. These electrons and their secondaries will then be lost from the avalanche and the multipactor will saturate. It seems reasonable to assume that the higher the SEY value, the greater will be the increase in electron number which accompanies 
the same increase in the width of the cloud. This explains why the saturation level increases continuously for increasing secondary emission yield, as found in numerical simulations carried out for relatively small values of SEY. A more detailed theoretical analysis of the mutual increase in the width and the number of electrons in the cloud is given in the Appendix.

One more critical value of the space charge parameter $\varepsilon$ can be found by analyzing the motion of the $T$ electrons. This motion is governed by Eq. (8), the solution of which, for given initial parameters (velocity $v_{0}$ and starting phase $\phi_{T}$ ), can be given as

$$
\xi=\left[-\frac{\varepsilon}{2} \tau^{2}+\left(v_{0}+\cos \phi_{T}\right) \tau\right]+\left[\sin \phi_{T}-\sin \left(\tau+\phi_{T}\right)\right],
$$

where $\tau=\phi-\phi_{T}$ denotes normalized time after the electron start. When the space charge parameter $\varepsilon$ is small, the two bracketed terms on the right-hand side of Eq. (17) can be seen as a drift with slowly varying velocity and a fast oscillation, respectively. The drift part is nothing but the motion of an electron in the dc retarded field with an effective initial velocity $v_{\text {eff }}=v_{0}+\cos \phi_{T}$. This represents a parabolic function of time where the maximum, $\xi_{d \max }=v_{\text {eff }}^{2} /(2 \varepsilon)$, decreases for increasing space charge parameter. Specifically, when $\varepsilon>v_{\text {eff }}^{2} /(2 \lambda)$, this maximum becomes less than the normalized gap width, $\lambda$. In the case of high order resonances, the normalized gap width is large [cf. Eqs. (10) and (12)] and the oscillatory part of the electron motion can be neglected when analyzing conditions related to gap crossings. This immediately implies that the $T$ electrons cannot cross the gap when the space charge parameter exceeds the value

$$
\varepsilon_{2}=\frac{\left(v_{0}+1\right)^{2}}{2 \lambda} \approx \frac{1}{2 T},
$$

where use has been made of the fact that, according to Eqs. (10) and (12), $\lambda \approx T\left(v_{0}+1\right) \approx T$. In this case, a considerable fraction of the electrons in the tail of the cloud returns back to the surface of emission. It should be emphasized that the threshold value $\varepsilon_{2}$ is in agreement with the saturation level of the multipactor found in the PIC simulations for $\sigma_{\max } \approx 1.75$ where single surface regimes were observed (see Fig. 11).

When $\varepsilon=\varepsilon_{2}$ and $\phi_{T}=0$, the solution given by Eq. (17) predicts electron return to the surface of emission in a time $4 T$, which equals an integer number of rf periods. This implies a possibility for returning electrons to contribute to the resonant motion of the single-surface multipactor ${ }^{25,26}$ and thereby to contribute to a further increase in the number of electrons. However, during the temporal interval $4 T$, the main part of the electron cloud, which is represented by the $R$ electrons, crosses the gap four times. This means that the trajectories of the $R$ and $T$ electrons are intersected and therefore the approximation of a fixed dc field in Eq. (8) is not valid. As a result, the single-surface resonance is not established when the threshold value for the space charge parameter $\varepsilon_{2}$ is reached. Correspondingly, an increase in the electron number is associated with an increase in the rate of electron losses and an establishing of the single surface resonance requires a further increase in the SEY value.

The situation changes when the space charge parameter exceeds the value $2 \varepsilon_{2}$. In this case, the solution given by Eq. (17) predicts that these electrons return to the surface of emission in a time less then $2 T$. The approximation of fixed $\varepsilon$ in Eq. (8) is then more justified and the realization of a stable single-surface resonance becomes possible for some fraction of the electrons. The establishing of a resonance promotes a reduction in electron losses and thereby allows further growth of the number of electrons even without an increase in the SEY value. According to the simple theory, ${ }^{25,26}$ a single-surface resonance is possible for a discrete series of the space charge parameter $\varepsilon$ :

$$
\varepsilon \approx \frac{1+v_{0}}{\pi p}
$$

where $p$ is an arbitrary integer number corresponding to the order of the single-surface resonance. This means that an increase in the space charge, which is caused by the formation of a single-surface resonance, makes it possible to establish one more single-surface resonance of reduced order. The new resonance also promotes a further increase in the space charge and thereby provides conditions for the appearance of still one more resonance of reduced order. This process continues as long as first order resonances are established and until the space charge parameter reaches approximately the value

$$
\varepsilon_{3}=\frac{1+v_{0}}{\pi} \approx \frac{1}{\pi} .
$$

Thus, the successive establishing of a series of single-surface resonances is accompanied by an increase in the space charge parameter from $2 \varepsilon_{2}$ to $\varepsilon_{3}$. This increase occurs without significant increase in the SEY value, in stark contrast with the sharp jump in the multipactor saturation level at the critical SEY value. The threshold values $2 \varepsilon_{2}$ and $\varepsilon_{3}$ are in agreement with the saturation level of the multipactor found in the PIC simulations at both sides of this jump (see Fig. 11). Finally, it should be noted that, within this simplified approach, the threshold value $\varepsilon_{3}$ was used in combination with Eq. (16) to estimate the number of multipacting electrons in the saturation stage in a dielectric-loaded acceleration structure. ${ }^{20}$ However, this estimate was applied independently of the value of the secondary emission yield, which is not quite correct, as demonstrated by our PIC simulations.

\section{CONCLUSIONS}

In the present work, a detailed study has been made of the saturation caused by the space charge effect of a multipactor discharge in a plane-parallel geometry. The saturation level of the number of multipacting electrons was found for a wide range of parameters using numerical PIC simulations. The saturation level and also the discharge type were shown to be sensitive to the value of the secondary emission yield of the electrode surfaces. For relatively weak secondary emission, the multipactor starts out as a conventional twosided multipactor discharge and preserves its two-sided na- 
ture at the saturation stage. However, when the secondary emission yield exceeds a certain threshold value, the multipactor transforms into a single surface phenomenon at the saturation stage. This transformation is accompanied by a drastic increase in the number of electrons in the multipactor discharge. The simulation results have been compared with predictions given by different theories of multipactor saturation. An analysis of the resonance stability and its perturbation by the space charge effect has been made in order to estimate the multipactor saturation level when the secondary emission yield is marginally larger than unity. On the other hand, when the secondary emission yield is significantly larger than the threshold value, the saturation level can be estimated using the single-surface multipactor theory. In intermediate cases, the multipactor includes both two- and onesided regimes and a simple estimate of the saturation level is not available.

\section{ACKNOWLEDGMENTS}

This work was partially supported by INTAS under Grant No. 06-1000024-9098 and by the Russian Foundation for Basic Research through Grant Nos. 07-02-00729a and 09-02-97024-a.

\section{APPENDIX: MULTIPACTOR SATURATION LEVEL}

In this appendix the dependence of the multipactor saturation level on the value of the secondary emission yield is analyzed. It is found that a partial compensation of the electron losses caused by a violation of the resonance conditions occurs for increasing SEY values. Consider first a conventional two-sided multipactor, which develops under the conditions of a classical resonance mode in situations where the SEY value is only slightly larger than unity. While the electron cloud oscillates between the plates, the space charge parameter $\varepsilon$ increases after each impact due to electron multiplication. Therefore the emission phases of the outermost electrons of the cloud are not repeated after each impact and the resonance conditions given by Eqs. (9)-(11) become violated. On the other hand, the interval between the phases of the outermost electrons becomes larger after each transit and the width of the electron cloud also increases. The following expressions represent the equations of motion and the initial conditions for the $F$ and $T$ electrons after $n$ transits between the plates:

$$
\begin{aligned}
& \mathrm{F}: \frac{d^{2} \xi}{d \phi^{2}}=\sin \phi+\varepsilon_{n}, \quad \xi\left(\phi=\phi_{F, n}\right)=0,\left.\quad \frac{d \xi}{d \phi}\right|_{\phi_{F, n}}=v_{0}, \\
& \mathrm{~T}: \frac{d^{2} \xi}{d \phi^{2}}=\sin \phi-\varepsilon_{n}, \quad \xi\left(\phi=\phi_{T, n}\right)=0,\left.\quad \frac{d \xi}{d \phi}\right|_{\phi_{T, n}}=v_{0} .
\end{aligned}
$$

It is tacitly assumed that the center of the cloud is not affected by the forces and that the motion of the $R$ electrons is described by Eq. (7). Using also the assumption that the secondary emission coefficient $\sigma$ is fixed and the same for all electrons of the cloud, the recurrence relation for the space charge parameter can be taken as

$$
\varepsilon_{n+1}=\sigma \varepsilon_{n} .
$$

When the emission phases of the outermost electrons are close to the emission phase $\phi_{R}$ of the central resonance, i.e.,

$$
\begin{aligned}
& \phi_{F}=\phi_{R}+\delta \phi_{F}, \quad \delta \phi_{F}<0, \quad\left|\delta \phi_{F}\right| \ll 1, \\
& \phi_{T}=\phi_{R}+\delta \phi_{T}, \quad 0<\delta \phi_{T} \ll 1,
\end{aligned}
$$

the following recurrence relations for $\delta \phi_{F, n+1}$ and $\delta \phi_{T, n+1}$ can be obtained from Eqs. (A1) and (A2):

$$
\begin{aligned}
\delta \phi_{F, n+1}= & \frac{1}{2 \cos \phi_{R}+v_{0}}\left[\delta \phi_{F, n}\left(T \sin \phi_{R}+v_{0}\right)\right. \\
& \left.+\frac{T \cos \phi_{R}}{2} \delta \phi_{F, n}^{2}-\varepsilon_{n} T^{2}\right], \\
\delta \phi_{T, n+1}= & \frac{1}{2 \cos \phi_{R}+v_{0}}\left[\delta \phi_{T, n}\left(T \sin \phi_{R}+v_{0}\right)\right. \\
& \left.+\frac{T \cos \phi_{R}}{2} \delta \phi_{T, n}^{2}+\varepsilon_{n} T^{2}\right] .
\end{aligned}
$$

As the number of electrons in the cloud grows, finally one of the outermost electrons impacts on a surface with an unfavorable phase and consequently the secondaries of this electron are not able to take part in the further development of the avalanche. Thus, the space charge becomes weaker and this allows further growth of the number of electrons until new outermost electrons are again lost.

The following scheme of recurrence calculations can be used to obtain a theoretical estimate of the threshold value $\varepsilon$, at which the saturation stage is reached. Assuming small initial deviations $\delta \phi_{F, 0} \ll 1$ and $\delta \phi_{T, 0} \ll 1$ together with a very small, but finite, value of $\varepsilon_{0} \ll 1$, the recurrence relations (A6) and (A7) can be solved after each transit taking into account Eq. (A3). The calculations are stopped as soon as at least one of the outermost electrons falls into unfavorable conditions. Specifically:

(I) The T electron is retarded (relative to the center of the cloud) so much that its transit time $\tau$ becomes much longer than what is typical for the considered classical mode: $\tau-T>2 \pi$; therefore this electron falls out of the studied electron cloud.

(II) the F electron crosses the gap too fast, so that the concomitant secondary electrons are released in starting phases that do not allow them to leave the surface, therefore these electrons are also lost.

In Fig. 12 the saturation levels obtained from numerical simulations carried out using the PIC code for various classical modes $k=3,5,7$ of the two-sided multipactor are compared with the theoretical estimates computed using the recurrence relations given by Eqs. (A3), (A6), and (A7) for $\sigma=\sigma_{\max }$ and $\phi_{R}=0$, with respect to conditions (I) and (II). In order to avoid any influence of the initial values $\varepsilon_{0}, \delta \phi_{F, 0}$, and $\delta \phi_{T, 0}$ on the theoretical estimates, the corresponding calculations were repeated many times and their results were 


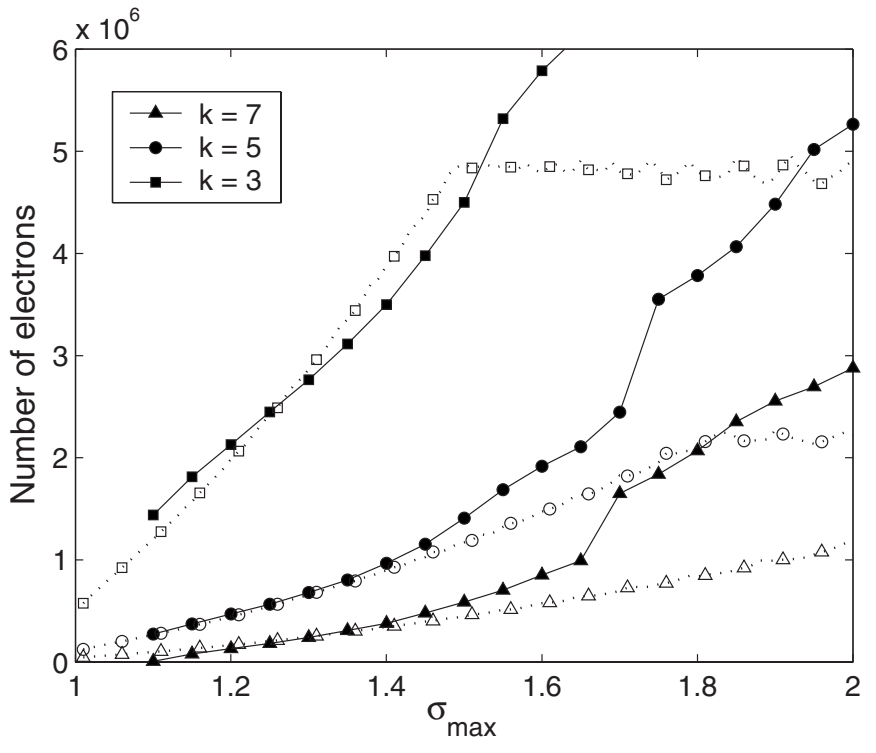

FIG. 12. Saturation levels of the number of electrons as functions of $\sigma_{\max }$. The solid line marked with squares corresponds to PIC numerical simulations carried out for the resonance mode $k=3 \quad(\lambda=10.37)$, the solid line marked with circles corresponds to PIC numerical simulations carried out for the resonance mode $k=5 \quad(\lambda=17.28)$, and the solid line marked with triangles corresponds to PIC numerical simulations carried out for the resonance mode $k=7 \quad(\lambda=24.19)$. The dotted lines marked, respectively, correspond to the theoretical estimates based on Eqs. (A3), (A6), and (A7).

averaged. It was found that condition (I) is stronger at lower $\sigma_{\max }$ values, whereas condition (II) is stronger at higher values of $\sigma_{\max }$. It is seen that the results of the PIC simulations are in good agreement with the theoretical estimates based on Eqs. (A3), (A6), and (A7) at low SEY values.

The small, but increasing, discrepancy observed for increasing values of $\sigma_{\max }$ (for $\sigma_{\max }<1.6$ ) should most likely be due to the simplification of the electron motion when the effect of the space charge on the center of the electron cloud is neglected, as discussed previously. It should also be noted that the discrepancies between the numerical and theoretical results become considerably larger for the SEY values at which the development of single surface regimes is detected and the model of the conventional two-sided multipactor cannot be applied.

More accurate theoretical estimates describing the saturation level of a mainly single surface multipactor must also take into account the influence of the actual SEY value. Thus, the increase in the saturation level after the jump can be explained as a compensation of the electron losses by the strong secondary emission. ${ }^{24}$ One possible method of analysis is presented in Ref. 13. However, in most of cases, due to the number of assumptions and simplifications usually made in a theoretical model, an analytical estimate is not accurate enough to predict the detailed multipactor properties and numerical simulations are then a more reliable approach for studying the properties of multipactor discharges.

${ }^{1}$ J. R. M. Vaughan, IEEE Trans. Electron Devices 35, 1172 (1988),

${ }^{2}$ R. A. Kishek, Y. Y. Lau, L. K. Ang, A. Valfells, and R. M. Gilgenbach, Phys. Plasmas 5, 2120 (1998).

${ }^{3}$ J. de Lara, F. Perez, M. Alfonseca, L. Galan, I. Montero, E. Roman, and D. Raboso, IEEE Trans. Plasma Sci. 34, 476 (2006).

${ }^{4}$ R. Udiljak, D. Anderson, M. Lisak, V. E. Semenov, and J. Puech, Phys. Plasmas 14, 033508 (2007).

${ }^{5}$ F. Perez, J. de Lara, L. Conde, M. Alfonseca, L. Galan, and D. Raboso, Simulation Modelling Practice and Theory 16, 1438 (2008).

${ }^{6}$ V. E. Semenov, E. Rakova, R. Udiljak, D. Anderson, M. Lisak, and J. Puech, Phys. Plasmas 15, 033501 (2008).

${ }^{7}$ M. A. Gusarova, V. I. Kaminsky, L. V. Kravchuk, S. V. Kutsaev, M. V. Lalayan, N. P. Sobenin, and S. G. Tarasov, Nucl. Instrum. Methods Phys. Res. A 599, 100 (2009).

${ }^{8}$ V. E. Semenov, E. I. Rakova, A. G. Sazontov, I. M. Nefedov, V. I. Pozdnyakova, I. A. Shereshevskii, D. Anderson, M. Lisak, and J. Puech, J. Phys. D 42, 205204 (2009).

${ }^{9}$ R. Kishek and Y. Y. Lau, Phys. Rev. Lett. 75, 1218 (1995).

${ }^{10}$ R. A. Kishek, Y. Y. Lau, and D. Chernin, Phys. Plasmas 4, 863 (1997).

${ }^{11}$ S. Riyopoulos, D. Chernin, and D. Dialetis, Phys. Plasmas 2, 3194 (1995).

${ }^{12}$ S. Riyopoulos, Phys. Plasmas 4, 1448 (1997).

${ }^{13}$ M. A. Gorshkova and V. E. Nechaev, Radiophys. Quantum Electron. 42, 967 (1999).

${ }^{14}$ L. G. Blyakhman, M. A. Gorshkova, and V. E. Nechaev, Radiophys. Quantum Electron. 43, 904 (2000).

${ }^{15}$ V. P. Gopinath, J. P. Verboncoeur, and C. K. Birdsall, Phys. Plasmas 5, 1535 (1998).

${ }^{16}$ L.-K. Ang, Y. Y. Lau, R. A. Kishek, and R. M. Gilgenbach, IEEE Trans. Plasma Sci. 26, 290 (1998).

${ }^{17}$ A. Valfells, J. P. Verboncoeur, and Y. Y. Lau, IEEE Trans. Plasma Sci. 28, 529 (2000).

${ }^{18}$ A. Coves, G. Torregrosa-Penalva, C. Vicente, B. Gimeno, and V. E. Boria, IEEE Trans. Electron Devices 55, 2505 (2008).

${ }^{19}$ J. G. Power, W. Gai, S. H. Gold, A. K. Kinkead, R. Konecny, C. Jing, W. Liu, and Z. Yusof, Phys. Rev. Lett. 92, 164801 (2004).

${ }^{20}$ L. Wu and L. K. Ang, Phys. Plasmas 14, 013105 (2007).

${ }^{21}$ O. V. Sinitsyn, G. S. Nusinovich, and T. M. Antonsen, Jr., Phys. Plasmas 16, 073102 (2009).

${ }^{22}$ A. Sazontov, M. Buyanova, V. E. Semenov, E. Rakova, V. Vdovicheva, D. Anderson, M. Lisak, J. Puech, and L. Lapierre, Phys. Plasmas 12, 053102 (2005).

${ }^{23}$ J. R. M. Vaughan, IEEE Trans. Electron Devices 36, 1963 (1989).

${ }^{24}$ A. Kryazhev, M. Buyanova, V. E. Semenov, D. Anderson, M. Lisak, J. Puech, L. Lapierre, and J. Sombrin, Phys. Plasmas 9, 4736 (2002).

${ }^{25}$ E. F. Vance, J. Appl. Phys. 34, 3237 (1963).

${ }^{26}$ V. E. Semenov, V. Nechaev, E. Rakova, N. Zharova, D. Anderson, M. Lisak, and J. Puech, Phys. Plasmas 12, 073508 (2005). 